\title{
Morfometria das fibras colágeno cardíaco em ratos sadios e diabéticos suplementados com vitamina $\mathrm{C}^{1}$
}

\author{
Thiago Guerra Diniz ${ }^{2}$, Hildebrando Gomes Benedicto ${ }^{3 *}$, Fernanda Rodrigues Agreste ${ }^{4}$, \\ Naianne Kelly Clebis ${ }^{5}$, Francisco Javier Hernandez-Blazquez ${ }^{4}$ e Pedro Primo Bombonato ${ }^{4}$
}

\begin{abstract}
Diniz T.G., Benedicto H.G., Agreste F.R., Clebis N.K., Hernadez-Blasquez F.J. \& Bombonato P.P. 2011. [Morphometry of the collagen fibers in healthy and diabetic rats treated with vitamin C.] Morfometria das fibras de colágeno cardiacas em ratos sadios e diabéticos suplementados com vitamina C. Pesquisa Veterinária Brasileira 31(Supl.1):1-6. Departamento de Morfologia, Instituto Biomédico, Universidade Federal Fluminense, Rua Professor Hernani Melo 101, Niterói, RJ 24210-130, Brazil. E-mail: hill@vm.uff.br

The increase of heart collagen fibers in diabetics is a well known fact, but the consequences are not defined. The aim was to quantify the cardiac collagen fibers in normal and diabetic rats treated with vitamin C. We selected 32 Wistar rats, 16 diabetic animals induced endovenously with streptozootocin, and 16 healthy animals, half of them, diabetics and normals, were treated with vitamin C for 90 days. After the experimental proceeding, the hearts were removed and processed accordingly to conventional protocol for optical microscopy and specific staining for collagen. The results showed that the diabetic rats presented increase in the number of cardiac collagen fibers, but the ones treated with vitamin $\mathrm{C}$ showed little accumulation of fibers. It could be concluded that treatment with vitamin $\mathrm{C}$ is important for the prevention of heart failure in diabetic animals.
\end{abstract}

INDEX TERMS: Heart, collagen, Diabetes mellitus, vitamin C.

\begin{abstract}
RESUMO.- 0 aumento do conteúdo de fibras colágenas no coração de diabéticos é um fato bastante conhecido, suas conseqüências ainda são objeto de estudo e causam certa controvérsia, portanto este trabalho objetivou estudar a variação na quantidade das fibras de colágeno cardíacas em animais normais e diabéticos tratados pela vitamina $\mathrm{C}$. Para isso foram selecionados 32 ratos Wistar, 16 diabéticos induzidos pela injeção endovenosa de estreptozootocina e 16 normais, sendo metade deles tratados com Vitamina C (diabéticos e normais) por um período de 90 dias. Após período experimental, os corações foram retirados e processados segundo protocolo convencional para microscopia
\end{abstract}

\footnotetext{
${ }^{1}$ Recebido em 24 de maio de 2011.

Aceito para publicação em 9 de setembro de 2011.

${ }^{2}$ Bolsista de Iniciação Científica do CNPq junto ao Departamento de Cirurgia, Faculdade de Medicina Veterinária e Zootecnia (FMVZ), Universidade de São Paulo (USP), Av. Prof. Orlando Marques de Paiva 87, Cidade Universitária, São Paulo, SP 05508-900, Brasil.

${ }^{3}$ Departamento de Morfologia, Universidade Federal Fluminense (UFF), Rua Hernani Pires de Mello 101, Centro, Niterói, RJ 24310-130, Brasil. *Autor para correspondência: hill@vm.uff.br

${ }^{4}$ Departamento de Cirurgia, FMVZ-USP, São Paulo, SP.

${ }^{5}$ Departamento de Morfologia, Universidade Federal do Rio Grande do Norte, Campus Universitário Lagoa Nova, Natal, RN 59072-970, Brasil.
}

óptica e coloração específica para colágeno. Os resultados mostram que animais diabéticos apresentam maior quantidade de fibras de colágeno cardíacas e que o tratamento com a vitamina $\mathrm{C}$ determinou um menor acúmulo na quantidade dessas fibras.

TERMOS DE INDEXAÇÃO: Coração, colágeno, Diabetes mellitus, vitamina $\mathrm{C}$.

\section{INTRODUÇÃO}

O coração apresenta um esqueleto de tecido conjuntivo que sustenta e dá inserção a musculatura. Os tipos principais de fibras de colágeno presentes no interstício do miocárdio são I, II e V, sendo predominante o tipo I. Seu aumento, no conteúdo total, tem sido evidenciado com o avanço da idade e em determinados estados patológicos, tal como o Diabetes mellitus.

Uma das principais causas de morte em diabéticos é a isquemia cardíaca devida a lesões das coronárias. Neles as plaquetas tendem a agregar-se mais facilmente por causa da glicolisação do colágeno, já que produzem um fator de crescimento que estimula a proliferação das células musculares dos vasos. Além disso, a glicolisação do colágeno 
aumenta a espessura da membrana basal, estreitando os vasos.

Sabe-se que o ácido ascórbico age como cofator na hidroxilação da prolina em colágeno, e que também se faz necessário na atividade funcional de fibroblastos e osteoblastos na formação de mucopolissacarídeos do tecido conjuntivo, do tecido ósseo, da dentina e do cemento intercelular dos capilares. A falta de vitamina $\mathrm{C}$ leva à formação de colágeno deficiente, fragilidade capilar e demora na cicatrização. Isto nos faz acreditar que ela tem papel não apenas construtor, mas também regulador na transcrição de algumas proteínas como principalmente das fibras colágenas, cuja síntese é influenciada pela presença ou ausência de agentes redutores. Essas fibras são os principais componentes do tecido conjuntivo presente na lâmina basal do tecido endotelial, podendo ser a ausência de vitamina $\mathrm{C}$ nos tecidos dos diabéticos, a razão da fragilidade capilar e cardiovascular desses organismos.

0 ácido ascórbico possui atividade anti-oxidante e combate a formação de radicais livres impedindo possíveis lesões nas coronárias, além disso, o mesmo tende a diminuir a formação de colágeno retardando assim a fibrose cardíaca, fatos estes ainda não devidamente comprovados. Por isso este foi o principal motivo que impulsionou este trabalho, que compara possíveis alterações do colágeno intersticial cardíaco em animais sadios e diabéticos suplementados com ácido ascórbico na expectativa de responder aos questionamentos a respeito da relação existente entre a formação de colágeno e o nível de ácido ascórbico.

\section{MATERIAL E MÉTODOS}

Para a realização deste experimento utilizamos 32 ratos Wistar, 16 machos e 16 fêmeas, que foram divididos em 4 grupos de 8 animais cada, sendo que 2 destes grupos eram constituídos por animais controle e os outros 2 grupos eram constituídos por animais diabéticos induzidos através da administração de $100 \mathrm{mg} / \mathrm{kg}$ de peso de Streptozotocina ${ }^{\circledR}$.

Um grupo dos animais controle e um grupo dos animais diabéticos induzidos receberam o tratamento com o Ácido Ascórbico através de gavagem, na dose de $20 \mathrm{mg}$ de Vitamina $\mathrm{C}$ por animal e através da administração de 1 grama de Vitamina C diluída em 1 litro de água oferecida nos bebedouros ad libitum.

Os animais diabéticos induzidos foram tratados com doses diárias de 0,1 UI de insulina NPH, para controle da glicemia, evitando-se um quadro agudo e fatal para os animais. 0 tratamento durou 3 meses e após este período os animais foram eutanasiados.

A eutanásia dos animais foi executada através da administração $0,5 \mathrm{ml}$ de tiopental sódico na diluição de $1 \mathrm{~g} / 20 \mathrm{ml}$ de soro fisiológico, via endovenosa, utilizando a veia da cauda. Após este procedimento procedemos à retirada dos corações através da abertura da caixa torácica dos animais.

Os corações foram imediatamente lavados e fixados em uma solução de formol a $10 \%$ por um período de 48 horas, a seguir foram submetidos ao tratamento histológico convencional, sendo cortados em 2 metades, abaixo das válvulas átrio-ventriculares, evidenciando os dois ventrículos esquerdo (VE) e direito (VD) e processados segundo a seqüência convencional: desidratação, diafanização e inclusão em blocos de Paraplast ${ }^{\circledR}$. No final obtivemos 32 blocos contendo meio coração em cada bloco. Foram selecionados duas lâminas por bloco, sendo dois cortes por lâmina, perfazendo um total de 128 amostras analisadas.
As lâminas foram coradas através da técnica do Picrosirius Red, Van Gieson+Fucsina-Paraldeído para evidenciar as fibras colágenas e, através da técnica Hematoxilina-Eosina (HE), para a padronização e identificação do material.

As lâminas foram observadas com auxílio do microscópio óptico Axioscópio Zeiss ${ }^{\circledR}$ e os resultados foram analisados em microcomputador com programa de morfometria específico (KS-400 Zeiss $^{\circledR}$ ), seguindo as etapas de captação da imagem, calibração do equipamento, engenharia da imagem (identificação da freqüência da cor do tecido estudado e a binarização da imagem), mensuração dos campos e a listagem dos dados.

Padronizamos a leitura e a mensuração de três campos de cada corte correspondente ao ventrículo direito e três campos, de cada corte referente ao ventrículo esquerdo, perfazendo um total de 768 leituras. Para efeito de mensuração foram desprezadas as áreas de tecido conjuntivo referentes ao epicárdio, endocárdio e margens das artérias ou veias (perivascular). Em seguida procedeu-se a análise e a discussão dos resultados.

Os dados foram agrupados segundo os grupos de origem, as áreas do coração das quais eles eram provenientes, o peso dos animais e a glicemias dos mesmos. Os dados adquiridos foram tratados estatisticamente em programas compatíveis com o ambiente Windows. Também utilizamos o teste de correlação de Pearson, com nível de significância de 5\%, para correlacionar as diferentes variáveis.

\section{RESULTADOS}

Os 32 ratos utilizados e divididos em 4 grupos foram monitorados durante todo o experimento (3 meses) quanto ao seu peso corpóreo e sua glicemia tendo-se obtido os seguintes resultados, demonstrados como segue no Quadro 1 e Quadro 2:

Quadro 1. Peso (g)/glicemia (g/dL) dos machos no momento da eutanásia

\begin{tabular}{ccccc}
\hline Machos & CTT & CNT & DTT & DNT \\
\hline 0 & $374,2 / 98$ & $472,6 / 110$ & $425,8 / 438$ & $488,2 / 442$ \\
1 & $426,4 / 100$ & $451,4 / 95$ & $468,8 / 435$ & $352,6 / 447$ \\
2 & $460,0 / 95$ & $349,8 / 104$ & $199,0 / 439$ & $369,0 / 461$ \\
3 & $461,0 / 87$ & $447,4 / 96$ & $376,4 / 433$ & $372,2 / 436$
\end{tabular}

Quadro 2. Peso(g)/glicemia(g/dL) das fêmeas no momento da eutanásia

\begin{tabular}{ccccc}
\hline Femeas & CTT & CNT & DTT & DNT \\
\hline 0 & $281,8 / 92$ & $253,8 / 95$ & $183,4 / 459$ & $227,6 / 537$ \\
1 & $252,6 / 95$ & $253,4 / 92$ & $146,4 / 452$ & $272,6 / 514$ \\
2 & $266,0 / 98$ & $273,2 / 94$ & $241,0 / 520$ & $217,6 / 533$ \\
3 & $243,6 / 91$ & $310,4 / 97$ & $264,6 / 465$ & $239,0 / 432$
\end{tabular}

\section{Grupos de animais controle tratados (CTT)}

Dentre as fêmeas deste grupo o peso médio foi de $241,68 \pm 16,58 \mathrm{~g}$, e sua glicemia média foi de $92,04 \pm 4,44 \mathrm{mg} / \mathrm{dl}$.

Já entre os machos, o peso médio foi de $357,63 \pm 56,26 \mathrm{~g}$. Sua glicemia média foi de $94,29 \pm 7,07 \mathrm{mg} / \mathrm{dl}$.

\section{Grupos de animais controle não tratados (CNT)}

As fêmeas deste grupo tiveram um peso médio de $248,24 \pm 20,43 \mathrm{~g}$. Sua glicemia média foi de $91,98 \pm 4,94 \mathrm{mg} / \mathrm{dl}$. 
Entre os machos deste grupo, o peso médio foi de $376,06 \pm 49,26 \mathrm{~g}$, e uma glicemia média de $429,86 \pm 12,62 \mathrm{mg} /$ dl.

\section{Grupos de animais diabéticos induzidos tratados (DTT)}

Neste grupo, as fêmeas tiveram um peso médio de $213,15 \pm 22,33$ gramas e uma glicemia media de $482,94 \pm 24,14 \mathrm{mg} / \mathrm{dl}$.

Contudo os machos deste grupo demonstraram um peso médio de 343,80 $\pm 36,93$ gramas. Já sua glicemia apresentou uma média de 429,86 $\pm 12,62 \mathrm{mg} / \mathrm{dl}$.

\section{Grupos de animais diabéticos induzidos não tratados (DNT)}

As fêmeas deste grupo apresentaram um peso médio de $232,85 \pm 20,07$ gramas. A glicemia média das fêmeas deste grupo foi de $532,17 \pm 21,93 \mathrm{mg} / \mathrm{dl}$.

Os machos apresentaram um peso médio de 364,38 $\pm 38,91$ gramas. Por fim a glicemia média deste grupo foi de $426,04 \pm 41,37 \mathrm{mg} / \mathrm{dl}$.

\section{Quantificação de colágeno}

As Figuras 1a e 1b identificam o tecido cardíaco através da coloração de Hematoxilina-Eosina (HE). Mas, para a descrição dos resultados obtidos na quantificação do colágeno cardíaco, valemo-nos dos 16 animais que tiveram os corações submetidos ao tratamento histológico convencional e corados através da técnica do Picrosírius Red demonstrada nas Figuras 1c e $1 \mathrm{~d}$.

Nesta quantificação pudemos verificar que entre o ventrículo direito dos animais CNT obtivemos uma concentra-

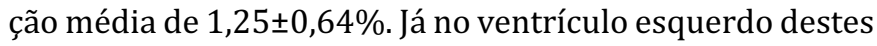
animais pudemos encontrar uma concentração média de $1,87 \pm 0,82 \%$.

No grupo CTT, o ventrículo direito dos animais apresentava uma concentração média de colágeno igual a 1,69 $\pm 0,63 \%$. No ventrículo esquerdo destes animais a concen-

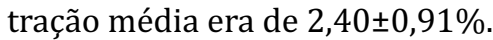

Entre o grupo DNT, o ventrículo direito dos animais

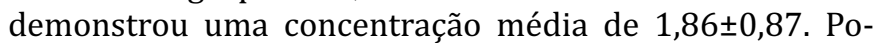
rém no ventrículo esquerdo a concentração média era de $2,44 \pm 1,01 \%$.

Por fim no grupo DTT o ventrículo direito apresentou uma concentração média de $2,27 \pm 0,89 \%$. Por fim, no ventrículo esquerdo a concentração média era de 2,35 0,98.

Após a obtenção destes dados foi possível correlacioná-los com o peso e a glicemia dos animais, medidos no momento da eutanásia, conforme mostram os Quadros 3 e 4. Esta correlação foi realizada através do teste de correlação de Pearson.

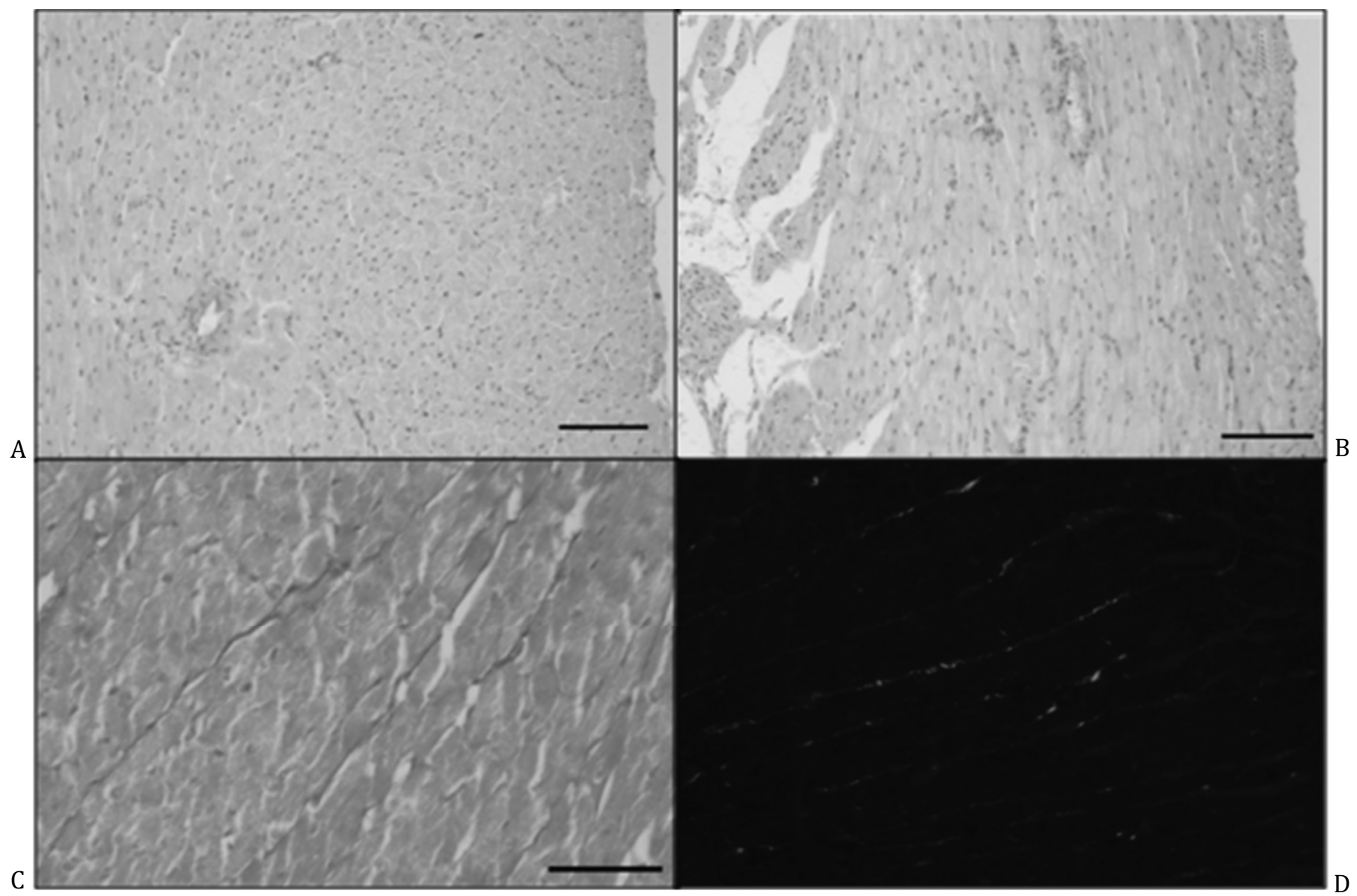

Fig.1. (a) Tecido muscular cardíaco de ratos do grupo CNT, VE. HE, barra = 100 $\mu$; (b) grupo DTT, VD. HE; (c) grupo CNT onde se evidenciam fibras de colágeno em vermelho. Picrossirius red, barra $=50 \mu \mathrm{m}$; (d) grupo CNT onde se evidenciam fibras de colágeno polarizadas nas cores amarela e vermelha (tipo I) verde (tipo III). Picrossirius red. 
Nestes testes pudemos verificar que a correlação existente entre a concentração de colágeno no Ventrículo Direito e o peso dos animais foi de $-0,720$ no grupo dos animais CTT, no grupo dos CNT a correlação é de 0,921. Entre os animais DTT esta correlação é de 0,076 e nos animais DNT apresentavam uma correlação de 0,754.

Quadro 3. Valores referentes à quantificação do colágeno cardíaco (\%) no ventrículo direito dos corações submetidos ao tratamento histológico convencional

\begin{tabular}{ccccccccc}
\hline Rato & VD1 & VD2 & VD3 & VD4 & VD5 & VD6 & $\begin{array}{c}\text { Média } \\
\text { VD }\end{array}$ & $\begin{array}{c}\text { Desvio } \\
\text { Padrão }\end{array}$ \\
\hline CNTF0 & 0,948 & 0,406 & 0,667 & 0,466 & 0,557 & 0,575 & 0,603 & 0,192 \\
CNTF1 & 0,265 & 0,361 & 0,306 & 2,086 & 1,143 & 1,499 & 0,943 & 0,756 \\
CNTM2 & 0,445 & 0,954 & 0,536 & 2,636 & 2,538 & 2,674 & 1,631 & 1,094 \\
CNTM3 & 1,369 & 2,459 & 1,283 & 1,827 & 1,536 & 2,346 & 1,803 & 0,501 \\
CTTF2 & 0,618 & 2,508 & 0,508 & 1,331 & 2,103 & 2,194 & 1,544 & 0,853 \\
CTTF3 & 1,570 & 2,814 & 1,719 & 2,033 & 2,191 & 3,560 & 2,315 & 0,749 \\
CTTM2 & 2,098 & 1,709 & 0,936 & 1,644 & 0,950 & 1,119 & 1,409 & 0,477 \\
CTTM3 & 1,387 & 1,046 & 1,405 & 1,100 & 1,592 & 2,319 & 1,475 & 0,461 \\
DNTF1 & 1,870 & 1,588 & 2,372 & 1,563 & 0,838 & 2,662 & 1,815 & 0,648 \\
DNTF2 & 1,062 & 1,834 & 0,718 & 1,165 & 0,760 & 1,097 & 1,106 & 0,401 \\
DNTM2 & 3,140 & 2,121 & 2,104 & 2,322 & 1,877 & 4,997 & 2,760 & 1,180 \\
DNTM3 & 0,805 & 1,100 & 1,951 & 0,920 & 1,529 & 4,140 & 1,741 & 1,250 \\
DTTF2 & 1,716 & 2,326 & 1,764 & 2,498 & 3,297 & 1,912 & 2,252 & 0,600 \\
DTTF3 & 2,247 & 1,393 & 1,024 & 0,711 & 1,288 & 1,597 & 1,377 & 0,526 \\
DTTM2 & 1,772 & 2,731 & 3,221 & 4,889 & 2,023 & 2,070 & 2,784 & 1,161 \\
DTTM3 & 1,388 & 4,870 & 2,969 & 2,216 & 1,621 & 2,939 & 2,667 & 1,261
\end{tabular}

Quadro 4. Valores referentes à quantificação do colágeno cardíaco (5) no ventrículo esquerdo dos corações submetidos ao tratamento histológico convencional

\begin{tabular}{ccccccccc}
\hline Rato & VE1 & VE2 & VE3 & VE4 & VE5 & VE6 & $\begin{array}{c}\text { Média } \\
\text { VE }\end{array}$ & $\begin{array}{c}\text { Desvio } \\
\text { Padrão }\end{array}$ \\
\hline CNTF0 & 1,006 & 0,942 & 0,641 & 2,649 & 3,043 & 4,169 & 2,075 & 1,169 \\
CNTF1 & 0,599 & 1,631 & 1,123 & 2,034 & 0,979 & 2,322 & 1,448 & 0,661 \\
CNTM2 & 1,216 & 1,751 & 1,126 & 2,272 & 2,259 & 2,805 & 1,905 & 0,795 \\
CNTM3 & 3,066 & 1,990 & 1,283 & 1,279 & 1,843 & 2,805 & 2,044 & 0,664 \\
CTTF2 & 1,627 & 2,879 & 2,634 & 2,884 & 1,816 & 3,315 & 2,526 & 0,867 \\
CTTF3 & 4,155 & 3,475 & 4,117 & 4,609 & 4,971 & 2,258 & 3,931 & 1,249 \\
CTTM2 & 1,246 & 1,377 & 1,797 & 1,033 & 3,488 & 2,795 & 1,956 & 0,770 \\
CTTM3 & 2,958 & 1,949 & 0,780 & 0,106 & 0,513 & 0,802 & 1,185 & 0,742 \\
DNTF1 & 5,999 & 1,995 & 3,277 & 1,629 & 0,740 & 1,850 & 2,582 & 1,302 \\
DNTF2 & 2,896 & 2,440 & 2,860 & 2,186 & 3,187 & 2,502 & 2,678 & 0,934 \\
DNTM2 & 3,422 & 2,754 & 3,004 & 1,548 & 2,002 & 2,265 & 2,499 & 0,908 \\
DNTM3 & 2,575 & 1,385 & 2,716 & 0,803 & 1,856 & 2,750 & 2,014 & 0,913 \\
DTTF2 & 5,688 & 1,791 & 2,561 & 1,364 & 2,870 & 4,273 & 3,091 & 1,237 \\
DTTF3 & 2,514 & 1,541 & 1,174 & 0,939 & 2,435 & 0,652 & 1,543 & 0,617 \\
DTTM2 & 3,016 & 1,768 & 1,118 & 4,392 & 3,940 & 3,581 & 2,969 & 1,128 \\
DTTM3 & 1,905 & 1,794 & 1,537 & 2,311 & 1,313 & 1,969 & 1,805 & 0,928
\end{tabular}

Na correlação entre a concentração do colágeno no ventrículo esquerdo e o peso dos animais, pudemos verificar que entre os animais do grupo CTT esta correlação é de $-0,862$. Nos animais CNT o valor desta correlação é 0,469 , entre os animais DTT a correlação é igual a -0,661 e entre os animais DNT esta correlação é de -0,748.

Realizou-se também a correlação entre a concentração de colágeno no ventrículo direito e a glicemia dos animais, e entre os animais do grupo CTT esse valor era de $-0,720$. Os animais CNT apresentaram uma correlação de 0,566 , os do grupo DTT obtiveram um valor de $-0,357$ e os animais DNT obtiveram uma relação igual a -0,576.

E por fim correlacionou-se também a concentração de colágeno no ventrículo esquerdo e a glicemia dos animais. Nesta correlação o grupo CTT apresentou um valor de 0,238, o grupo CNT teve uma correlação igual a 0,397. Já entre os animais diabéticos induzidos, o grupo DTT teve uma correlação de 0,447 e o grupo DNT teve uma correlação de 0,874 .

Para efeito de comparação, determinamos a quantidade de fibras colágenas nas áreas perivasculares, desprezadas para fins de resultados e discussões, onde encontramos os seguintes resultados: animais do grupo CNT apresentaram $4,05 \%$ de fibras colágenas, o grupo CTT apresentou 4,16\% de fibras, o grupo DNT 7,96\% e o grupo DTT 6,39\%; fato este que denota uma acumulação de fibras colágenas perivasculares segundo a presença do diabetes.

\section{DISCUSSÃO E CONCLUSÕES}

Os trabalhos morfológicos que analisam aspectos quantitativos têm teor importante pois oferecem precisão e acurácia no estabelecimento de conceitos ou no subsidiamento de conhecimentos morfológicos.

Nossas observações quanto ao comportamento do tecido conjuntivo cardíaco são concordantes com aquelas descritas por Gay \& Miller (1978), Nickel et al. (1981), Dyce et al. (1987) Montes \& Junqueira (1988), Weber (1989), Junqueira \& Carneiro (1995); no entanto vale ressaltar que as referidas citações são fontes descritivas, ora das estruturas em estudo, ora do órgão como um todo e mesmo de importância funcional, mas em nenhum momento, fazem menção a aspectos quantitativos, tais como os por nós estudados.

Conforme Medugorac (1980), Borg \& Caufield (1981), Medugorac (1982), Medugorac \& Jacob (1983), Robinson et al. (1983), Weber (1989) e Janicki et al. (1995), Legrice et al. (1995), Rossi et al. (1998), Prado et al. (2003), Benedicto \& Bombonato (2003) e Leite et al. (2004), o colágeno está presente no miocárdio em relativa pequena quantidade, 2 a $4 \%$ do total do volume miocárdico, e é a proteína estrutural mais abundante da rede de tecido conjuntivo. Estão entremeados, em grande quantidade, entre o coração e entre a aorta e a artéria pulmonar, podem ser encontrados também entre a musculatura atrial e ventricular e entre pacotes de miócitos e envolvendo os miócitos, individualmente (Macchiarelli et al. 2002). São importantes pré-requisitos para o bom funcionamento do coração, sendo responsáveis pelo mecanismo ventricular, contração e relaxamento cardíaco. Pudemos verificar em nosso traba- 
lho que a concentração de colágeno ventricular é proporcional à variação de colágeno em cada segmento, ventrículo direito ou esquerdo, conforme citado pelos autores acima e, também, pode variar com a idade, estados patológicos e fisiológicos compensatórios, que são temporários.

Sobel \& Marmorston (1956), Clausen (1963), Wezár (1964); Medugorac (1982), Dolber \& Spach (1987), e Debessa et al. (2001) em seus trabalhos demonstraram que a idade é um fator estimulante da deposição de colágeno cardíaco, pois quanto mais velho é o animal maior a quantidade colágeno cardíaco e, conseqüentemente, maior o seu risco de morte devido a algum acidente cardiovascular. Além disso, os trabalhos de Becker e Anderson (1983), Thomas (1987) e Debessa et al. (2001) mostram e reforçam a idéia que há modificações na quantidade e qualidade do colágeno cardíaco determinadas por um remodelamento muscular cardíaco; no entanto Burgess (2001) refere-se a fibrose cardíaca como sendo o fator determinante das alterações cardíacas. No nosso trabalho obtivemos informações complementares as destes autores, pois verificamos que houve aumento da quantidade de colágeno cardíaco em animais diabéticos e, ainda, encontramos a influência do peso na deposição do colágeno cardíaco, ou seja, os animais mais pesados tinham uma maior concentração de colágeno cardíaco. Outra informação pertinente se refere ao tratamento com a vitamina $C$, pois, animais tratados tiveram menor índice de acumulação de colágeno que animais não tratados, fato esse importantíssimo quando da decisão de tratar ou não os animais.

Já Rubler et al. (1972), Arduino (1973), Regan et al. (1974), Kannel \& Mcgee (1979), Koltai et al. (1984), Chen (2000), Bell (2003) e Gilles (2003) são autores que descreveram a etiologia, a patologia e a sintomatologia do Diabetes mellitus, que foi a doença de estudo deste trabalho. Goodfellow (1997), Neckar et al. (2001), referem-se ao acúmulo de colágeno em corações diabéticos ser o principal fator pela alteração funcional do miocárdio. Silva (1995) descreve que o diabetes pode desencadear a Miocardiopatia dilatada e associado aos estudos de Pogatsa (1979) concordam que há aumento no acúmulo de colágeno cardíaco. Todos eles foram enfáticos ao relatarem que os indivíduos com esta endocrinopatia estão mais susceptíveis a doenças do sistema cardiovascular e a alterações do tecido conjuntivo cardíaco. Estas afirmações são compatíveis com os achados em nosso trabalho, pois concluímos que o diabetes, $\mathrm{o}$ aumento do peso e as altas taxas glicêmicas foram fatores determinantes também na acumulação de colágeno e que a suplementação de vitamina $C$ promove a diminuição desse acúmulo, concordando com o trabalho de Faria et al. (2004).

Lewis et al. (1990), Pond et al. (1995), Lindsay et al. (1998) e Faria et al. (2004) foram fontes utilizadas para descrever as funções, constituição e fontes de obtenção do ácido ascórbico, que foi o tratamento utilizado nos animais deste estudo.

Segundo Lewis et al. (1990) a vitamina C estimula a síntese do colágeno através da ativação da enzima Colágeno Prolina Hidroxilase, contida nos fibroblastos. Porém em nosso trabalho encontramos bases para dizer que o tra- tamento com a vitamina $\mathrm{C}$ impediu um maior aumento da acumulação de colágeno se comparados animais tratados e não tratados, mas não foi tão efetiva quanto esperávamos. Talvez essa eficiência parcial seja pelo tempo de tratamento e indução dos animais. Fato este importante na continuação de estudos com uso da vitamina $\mathrm{C}$ em doses variadas e por tempo mais prolongado.

Em face aos resultados apresentados e os argumentos discutidos viemos por concluir que houve aumento na concentração de fibras colágenas nos animais diabéticos se comparados aos animais normais e que o tratamento com a vitamina C impediu um maior aumento da acumulação de colágeno comparando animais tratados e não tratados, mas não foi tão efetiva do ponto de vista quantitativo. 0 peso e a glicemia, também, influenciaram positivamente na deposição do colágeno, ou seja, quanto maior o peso ou a glicemia maior a quantidade de colágeno cardíaco depositada.

\section{REFERÊNCIAS}

Arduino F. 1973. Diabetes e suas Complicações. 2ª ed. Guanabara Koogan, Rio de Janeiro, p.1-4.

Becker A.E. \& Anderson R.H. 1983. Cardiac pathology, p.54. In: Ibid. (Eds), An Integrated Text and Colour Atlas. Churchill Livingstone, Edinburgh.

Bell D.S.H. 2003. Diabethic cardiomyopathy. Diabetes Care 26:2433-2441.

Benedicto H.G. \& Bombonato P.P. 2003. Quantificação de tecido conjuntivo do músculo cardíaco de cães. Braz. J. Vet. Res. Anim. Sci. 40(2);108-116.

Borg T.K. \& Caufield J.B. 1981. The collagen matrix of the heart. Fed. Proc. 40(7):2037-2041.

Burguess M.L., Mccrea J.C. \& Heather L.H. 2001. Age-associated changes in cardiac matrix and integrins: mechanisms of ageing and development 122(15):1739-1756.

Clausen B. 1963. Influence of age on chondroitin sulfates and collagen of human aorta, myocardium and skin. Lab. Invest. 12:538-542.

Chen S., Evans T., Mukherjee K., Karmazyn M. \& Chakrabarti S. 2000. Diabetes-induced myocardial structural changes: Role of endothelin-1 and its receptors. J. Mol. Cell Cardiol. 32(9):1621-1629.

Dyce K.M., Sack W.O. \& Wensing C.J.G. 1987. Tratado de Anatomia Veterinária. Guanabara Koogan, Rio de Janeiro, p.145-160.

Debessa C.R.G., Maifrino L.B.M. \& Souza R.R. 2001. Age related changes of the collagen network of the human heart. Mechanisms of Ageing and Development 122:(13)1049-1058.

Dolber P.C. \& Spach M.S. 1987. Thin collagenous septa in cardiac muscle. Anat. Rec. 128(1):45-55.

Faria H.G., Stabille S.R. \& Cassaro L. 2004. Efeitos da ingestão de água suplementada com vitamina $\mathrm{C}$ no desempenho de ratos submetidos à condição de estresse. Arqs Apadec, Maringá, 8(8):1-6.

Gay S. \& Miller E.J. 1978. Collagen in the physiology and pathology of connective tissue. Gustav Fischer, New York, p.1-106.

Giles T.D. 2003. The patient with diabetes mellitus and heart failure: At risk issues. Am. J. Med. 115(8A):107S-110S.

Goodfellow J. 1997. Microvascular heart disease in diabetes mellitus. Diabetologia 40(2):130-133.

Janicki J.S., Brower G.L., Henegar J.R. \& Wang L. 1995. Molecular and subcellular cardiology: Effects of structure and function. Plenum Press, New York, p.239-244.

Junqueira L.C. \& Carneiro J. 1995. Histologia Básica. 8ª ed. Guanabara Koogan, Rio de Janeiro, p.69-93,170-173,187-189.

Kannel W.B. \& McGee D.L. 1979. Diabetes and cardiovascular disease: The Framingham study. J. Am. Med. Assoc. 241(19):2035-2038.

Koltai M.Z., Balogh I., Wagner M. \& Pogatsa G. 1984. Diabetic myocardial alterations in ultrastructure and function. Exp. Pathol. 25(4):215-221. 
Legrice I.J., Smaill B.H., Chai L.Z., Edgar S.G., Gavin J.B. \& Hunter P.J. 1985. Laminar structure of the heart: Ventricular myocite arrangement and connective tissue architecture in the dog. Am. J. Physiol., Heart Circul. Physiol. 269(2):H571-H582.

Leite E.P., Bombonato P.P., Carneiro-Silva F.O., Benedicto H.G. \& Santana M.I.S. 2004. Morfometria do tecido conjuntivo do coração de eqüinos PSI. Braz. J. Vet. Anim. Sci. 41(3):162-168.

Lewis L.D., Morris M.L. \& Sand M.S. 1990. Small Animal Clinical Nutrition. Vol.3. $3^{\text {rd }}$ ed. Mark Morris Institute, Topeka, Kansas, p.77-100.

Lindsay R.M., Jamieson N.S.D., Walker S.A., McGuigan C.C., Smith W. \& Baird J.D. 1998. Tissue ascorbic acid and polyol pathway metabolism in experimental diabetes. Diabetologia 41:516-523.

Macchiarelli G., Ohtani O., Nottola S.A., Stallone T., Camboni A., Prado I.M.M. \& Motta P.M. 2002. A micro-anatomical model of the distribuition of myocardial endomysial collagen. Histol. Histopathol. 17(3):699-706.

Meducorac I. 1980. Collagen content in different areas of normal and hyperthophied rat myocardium. Cardiovascular Res. 14:551-557.

Meducorac I. 1982. Caracterization of intramuscular collagen in the mammalian left ventricule. Basic Res. Cardiol. 77:589-598.

Meducorac I. \& Jacob R. 1983. Caracterization of left ventricular collagen in the rat. Cardiovascular Res. 17:15-21.

Montes G.S. \& Junqueira L.C.U. 1988. Histochemistry. Vol.2. Springer Verlag, Berlin, p.41-54.

Neckar J., Kolat F., Stetka R., Sczielgelhoffer A. \& Stik J. 2001. Ventricular arrhytmias following coronary artery occlusion in rats : Is the diabetic heart less or more sensitive to ischemia. Basic Res. Cardiol. 96(2):160168.
Nickel R., Schummer A. \& Seiferle E. 1981. The Anatomy of Domestic Animals. Vol.3. Verlag Paul Parey, Hamburg, p.15-38.

Pond W.G., Church D.C. \& Pond K.R. 1995. Basic Animal Nutrition and Feeding. John Willey and Sons, New York. 70p.

Pogatsa G., Bihari-Varga M. \& Szinay G. 1979. Effect of diabetes therapy on the myocardium in experimental diabetes. Acta Diabetal Lab. 16:129.

Prado I.M.M., Liberato J.A.D., Miranda-Neto M.H., Molinari S.L., Stallone T., Macchiarelli G. \& Motta P.M. 2003. Distribuição of collagen fibers in the aggregated lymphoid follicles of swine terminal ileum. Annals of Anatomy 185(1):73-80.

Regan T.J., Extinger P.O. \& Kahn M. 1974. Altered myocardial function and metabolism in chronic diabetes mellitus without ischemia in dogs. Circulation Res. 35(2):222.

Robinson T.H., Cohen-Gould L. \& Factor S.M. 1983. Skeletal framework of mammalian hearth muscle. Lab. Invest. 49(4):482-498.

Rossi M.A., Abreu M.A. \& Santoro L.B. 1998. Connective tissue skeleton of the human hearth: A demonstration by cell-maceration scanning electron microscope method. Circulation 66:183-194.

Rubler S., Dlugash J., Yuceoglu Y.Z., Kumral T., Branwood A.W. \& Grishman A. 1972. New type of cardiomyopathy associed with diabetic glomerulosclerosis. Am. J. Cardiol. 30(6):595-602.

Thomas W.P. 1987. Myocardial diseases of the dog, p.117-156. In: Bonagura J.D. (Ed.), Cardiology. Churchill Livingstone, New York.

Weber K.T. 1989. Cardiac interstitium in health and disease: The fibrilar collagen network. J. Am. Coll. Cardiol. 13(7):1637-1652.

Wezár F. 1964. The stages and consequences of aging of collagen. Gerontologia 9:209-221. 